\title{
Origin of the Buccal Branch of Facial Nerve and Anastomosis of the Facial Nerve Branches
}

\author{
Yousif I Eltohami ${ }^{1, *}$, Shiang-Fu Huang ${ }^{2}$, and Ahmed M Suleiman ${ }^{3}$ \\ ${ }^{1}$ Assistant professor of Oral \& Maxillofacial Surgery, University of Khartoum, Sudan \\ ${ }^{2}$ Professor of Otolaryngology, Head and Neck Surgery, Chang Gung Memorial Hospital, Taiwan \\ ${ }^{3}$ Professor of Oral \& Maxillofacial Surgery, University of Khartoum, Sudan
}

*Corresponding author: Eltohami YI, Assistant professor of Oral \& Maxillofacial Surgery, University of Khartoum, Sudan, E-mail: yof88@hotmail.com

Received: 14 Aug, 2019 | Accepted: 23 Aug, 2019 | Published: 02 Sep, 2019

Citation: Eltohami YI, Huang SF, Suleiman AM (2019) Origin of the Buccal Branch of Facial Nerve and Anastomosis of the Facial Nerve Branches. J Clin Case Stu 4(2): dx.doi.org/10.16966/2471-4925.191

Copyright: (C) 2019 Eltohami Yl, et al. This is an open-access article distributed under the terms of the Creative Commons Attribution License, which permits unrestricted use, distribution, and reproduction in any medium, provided the original author and source are credited.

\section{Abstract}

Background: In parotid surgery, the extratemporal course of the Facial Nerve (FN) is very variable. Familiarity with these variations in the branching patterns is extremely crucial in successful dissection and preservation of the nerve. The present study aimed to describe the origin of the buccal branch of facial nerve and anastomosis of the facial nerve branches.

Methodology: A total of 90 facial nerves were dissected, [40 cadavers (bilateral) and 10 patients (unilateral)]. Out of these 50 cases, 41 were males ( 37 cadavers and 4 patients) and 9 were females ( 3 cadavers \& 6 patients). Forty-six (51\%) were left and 44 (49\%) were right facial nerves. Photographs of intra-parotid distribution of each facial nerve were taken during dissection.

Results: In $78.8 \%$ of the cases, the buccal branch originated mainly from the lower division. Anastomotic connections were present between the buccal nerve and lower division in $36.67 \%$ of the cases, between the upper and lower divisions in $33.3 \%$ of the subjects, between the main trunk \& lower division in $7.78 \%$ of the cases and between the buccal branch \& the upper division in $2.2 \%$ of the cases.

Conclusion: Majority of the buccal branches originated from the cervicofacial division and most of the anastomotic interconnections were identified between the lower and upper division. Anatomical variations in the origin and anastomosis can shed more light on the different consequences of facial paralysis that may encounter after parotid field surgery.

Keywords: Facial nerve; Origin of buccal branch; Anastomosis

\section{Introduction}

The buccal branch was described as a single or double in number. It goes horizontally to supply the region of the cheek. The buccal branch has an intimate contact with the parotid duct. The branches could be divided into superior and inferior parts. Superior branches go below the zygomaticus major and levator labii superioris. Also give innervation to zygomaticus minor, levator labii superioris alaeque nasi and levator angulii oris. In other hand, inferior ones supply the buccinators and orbicularis oris [1]. Patients with facial paralysis may experience tremendous psychosocial distress about their conditions. Poor self-image and difficulty interacting with peers and family members can be frustrating [2].

Kwak $\mathrm{HH}$, et al. studied the branching patterns of the facial nerve among Korean population. A micro dissection was performed on 30 half-heads, and the FN trunks and branches were exposed. According to the origin of the buccal branches, the investigators classified the branching patterns of the FN into four categories. In type I (13.8\% of cases), the buccal branches arose from the two main divisions of the trunk but not from other branches of the facial nerve. In type II (44.8\% of cases), the buccal branches arising from the two main divisions were interconnected with the zygomatic branch. In type III (17.3\% of cases), the marginal mandibular branch sent nerve twigs to the buccal branch, which originated from the upper and lower divisions. In type IV (17.3\% of cases), the nerve twigs from the zygomatic and marginal mandibular branches merged to the buccal branch arising from the two main divisions [2]. The present study aimed to describe the origin of the buccal nerve branch of facial nerve and anastomosis of the facial nerve branches, because of the extreme variability of the anatomy of parotid region and functional significance of the course of topography of the buccal nerve branch and it is anastomosis with other facial nerve branches.

\section{Methodology}

A total of 90 facial nerves were dissected, [40 cadavers (bilateral) and 10 patients (unilateral)]. Out of these 50 cases, 41 were males (37 
cadavers and 4 patients) and 9 were females ( 3 cadavers \& 6 patients). Forty-six (51\%) were left and 44 (49\%) were right facial nerves. The study was conducted in Khartoum Teaching Dental Hospital and the Dissection Rooms at the Department of Anatomy, medical campuses of different universities and Bashair mortuary in Khartoum state, Sudan. Ethical approval was obtained from the University of Khartoum Faculty of Dentistry, Ethical Committee Review Board, research unit at Khartoum Teaching Dental Hospital and a signed written consent was obtained from the patients. The samples were collected by equation:

$$
\mathrm{n}=4\left[\left(\pi \_ \text {plan }\left(1-\pi \_ \text {plan }\right)\right) / \omega^{\wedge} 2\right] z_{-}(1-\alpha / 2)^{\wedge} 2
$$

\section{Where,}

n-sample size

$\pi \_$plan-The anticipated population proportion

$\alpha$-The level of significance

$z_{-}(1-\alpha / 2)$-The value from the normal distribution related to and representing the confident interval.

$\omega$-The width of the confidence interval.

Depending on the previous study [2] for this study, the $\omega$ anticipated population proportion $\pi \_$plan was equal to 36.7 . The sample size was 90 cases.

Patients with history of previous surgical operations in the parotid region, subjects with history of maxillofacial trauma which damaged the facial nerve and previous damaged intraparotid facial nerve in cadaveric dissection were excluded.

Data were entered in a computer master sheet using SPSS version 16. All statistical analysis was set at $95 \%$ confidence level, 0.2 the width of the confidence interval and the level of significance, 0.05 . Descriptive statistics were conducted for variables: gender and site. The Wilcoxon signed-rank test declared no statistical significance between both sides of the face regarding the origin of the buccal branch.

Procedure: In all cases an incision was made anterior to the tragus of the ear and extending down below the lobule of the ear. An inverted $S$-shaped incision was extended behind the ear and inferio-posterior to the angle of the mandible. In case of anterior parotid lesion the incision was extended further down the neck. After elevating the flaps, the anterior border of sternocleidomastoid muscle was dissected to the mastoid process. The posterior belly of digastric muscle was visualized. The cartilaginous portion of external auditory canal was dissected to the bony portion. So in an orthograde approach the facial nerve was identified using the two standard anatomic landmarks (the tragal pointer and the posterior belly of digastric muscle). The main trunk of the facial nerve was visualized and dissected peripherally distal to the stylomastoid foramen and tracing of its branches was carried out. For the retrograde approach the buccal branch was used as a guide to reach the main trunk.

Following dissection, the pattern of the buccal branch and anastomosis of the facial nerve branches were photographed.

\section{Results}

\section{Origin of the buccal nerve branch was found as follows:}

The majority of the buccal branches arose from the lower division in $78.8 \%$ (Figure 1) and both divisions in $21.1 \%$ cases (Figure 2). There were no cases that the buccal branches arise from the upper division alone.

\section{Anastomotic connections of facial nerve were found as follow:}

Between the buccal nerve branch and both lower \& upper divisions in $36.67 \%$ and $2.2 \%$ of the cases respectively.

In $33.3 \%$ of the cases anastomosis between the upper and lower divisions of the facial nerve was identified and was found between the main trunk \& lower division in $7.78 \%$ of the cases (Figures 3-5).

\section{Discussion}

Anatomical variabilities in the origin and anastomosis of the buccal branch were reported as the commonest variations in the topography of the extracranial facial nerve [3]. A noteworthy, that transient facial paralysis has been documented in about $20-55 \%$ and permanent damage was reported in $1-2 \%$ of the subjects underwent

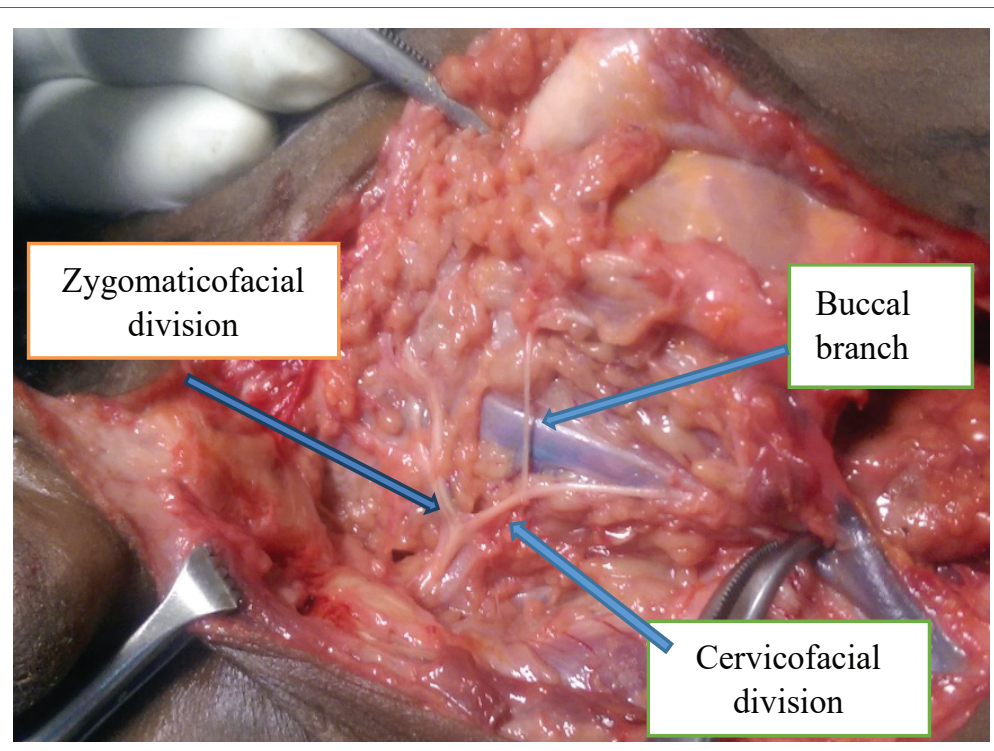

Figure 1: Origin of the buccal nerve branch from lower division. 


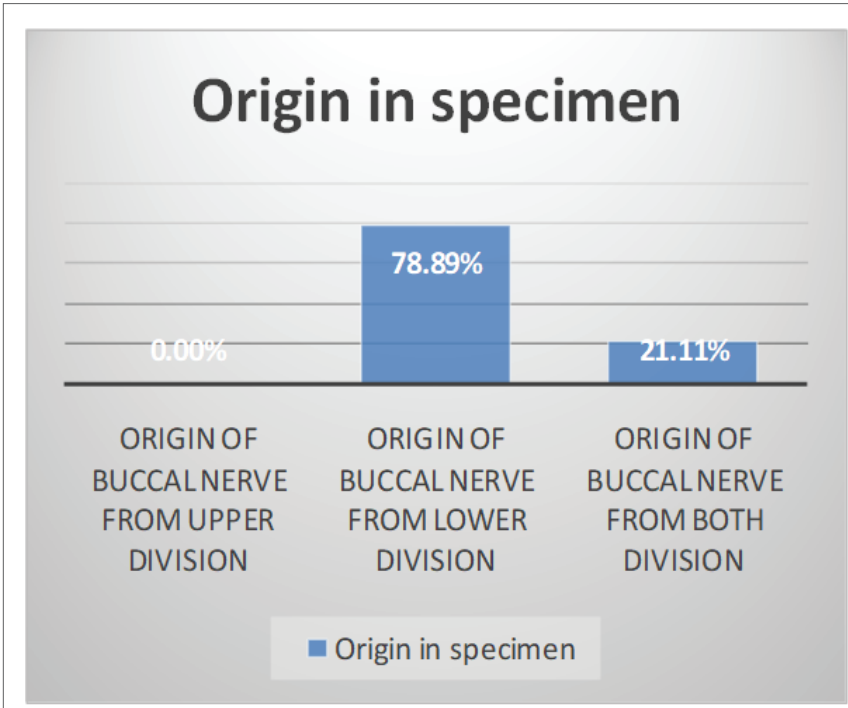

Figure 2: Distribution of the buccal nerve branch origin.

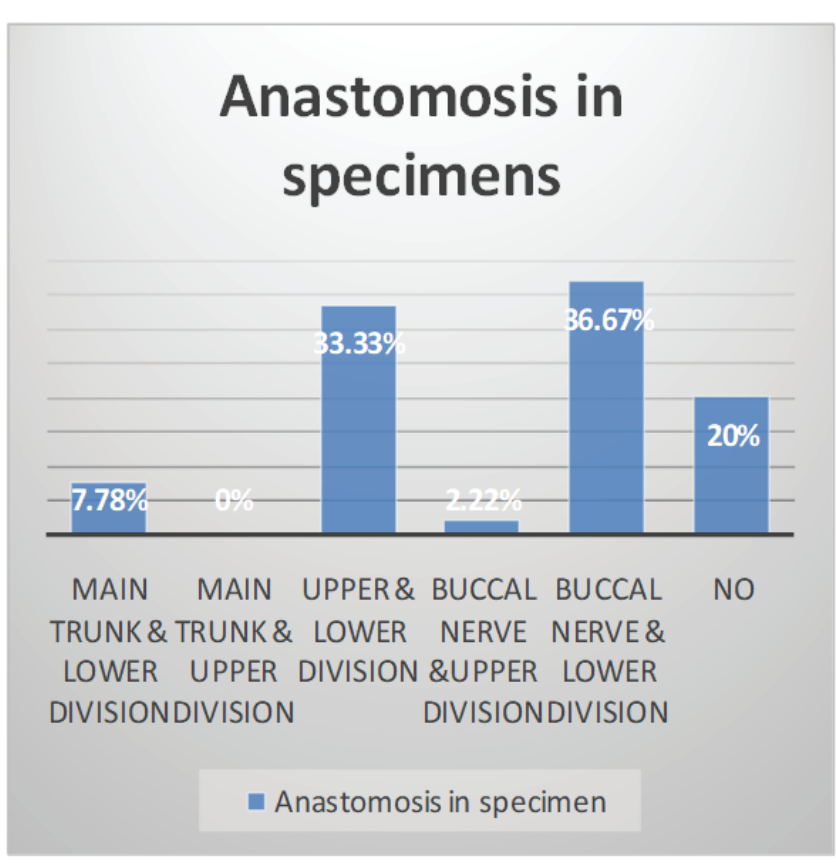

Figure 3: Anastomosis of the main trunk with the facial nerve divisions.

surgical excision of the parotid tumors [4]. So the arborization pattern topography of the FN is an utmost highly complicated anatomical variation, which indicates the extreme intimate familiarity with this anatomical complexity during performing surgeries in this field [5].

There were many studies performed on the origin of the buccal nerve branch. In the present study the frequency of origin of the buccal nerve branch from the lower division was $78.8 \%$ of the cases. This finding was in agreement with the studies carried by Davis RA, et al. and Farooq Ahmed, et al. [6,7] which varied from $52.6 \%$ to $84 \%$ respectively.

There was no case of buccal nerve branch originating from the upper division. In contrast to the present findings, Davis RA, Farooq Ahmed,

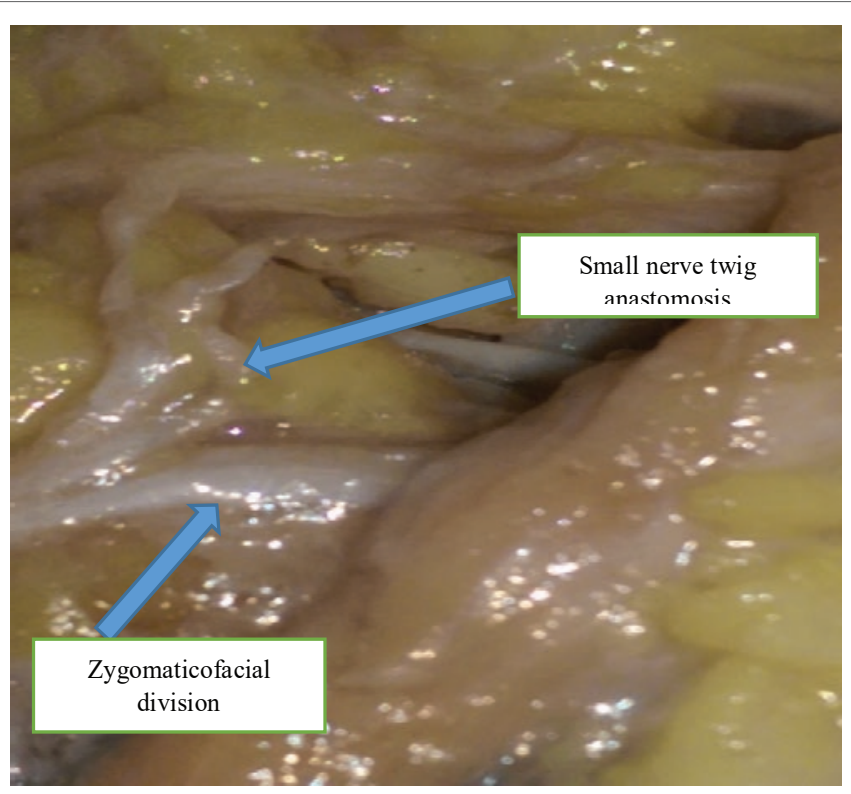

Figure 4: One trunk and Small nerve twig anastomosis with the zygomaticofacial division (left side).

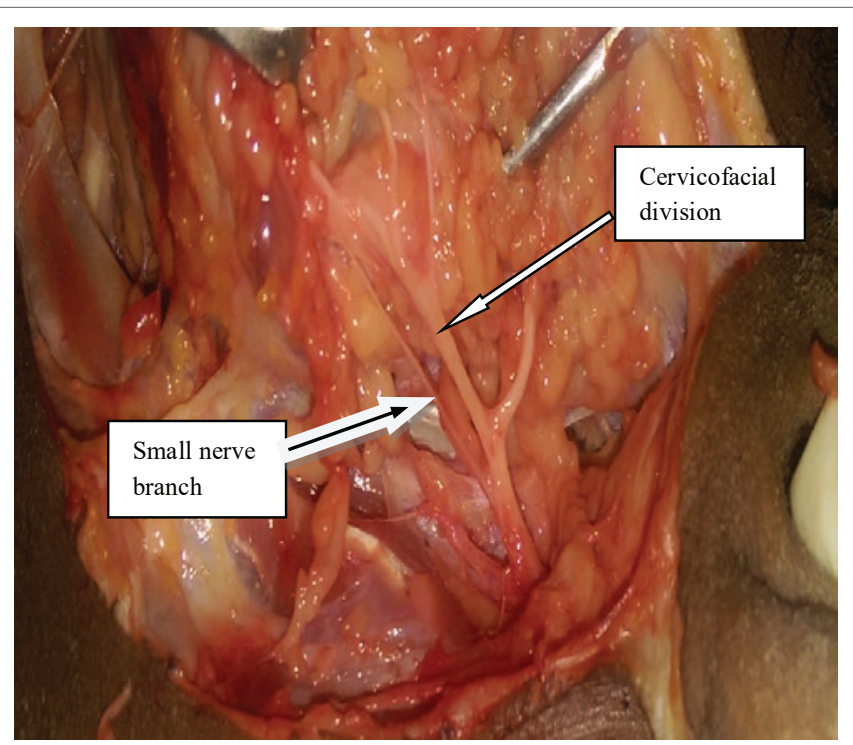

Figure 5: One main trunk and Small nerve branch anastomosis with the cervicofacial division (left side).

Kopuz C, et al. [6-8] found the buccal nerve branch originated from the upper division in $20 \%, 18 \%$ and $42.1 \%$ of the cases respectively.

In the present study the prevalence of buccal nerve branch originating from the both divisions was $21.1 \%$, which was different from the findings by Davis RA, Katz AD, and Farooq Ahmed, et al. $4 \%$, $4 \%$ and $5.2 \%$ respectively $[6,7,9]$. A higher different finding $36 \%$ of the cases were found by Kopuz C et al. [8].

Anastomotic connections were identified between the buccal nerve and lower division in $36.67 \%$, between the upper and lower divisions in $33.3 \%$, and between the buccal nerve \& upper division in $2.2 \%$ of the cases. Farooq Ahmed, et al. [7] identified anastomotic interconnections between the buccal nerve and lower division in $8.7 \%$ 
of the cases, between the upper and lower divisions in $8.7 \%$ of the cases and between the buccal nerve \& upper division in 19.2\% of the cases. In contrast to the findings by Kopuz C, et al [8] where no anastomotic connections between the buccal nerve branch and divisions of the facial nerve were found. Additionally, there is a previous study explored a significant anastomosis between buccal, temporal and inferior division branches in two third of the subjects [9]. Yang HM, et al. documented an extremely variable interconnection between the FN branches in all dissected specimens. [10].

Farooq Ahmed, et al. [7] reported no case of anastomotic interconnections between the main trunk \& the lower division. While Kopuz C, et al. [8] identified this type of anastomosis in $8 \%$ of the cases, which similar to this study finding $7.78 \%$.

The present study was in agreement with Farooq Ahmed, et al. [7] findings in that there was no anastomosis identified between the main trunk \& the upper division. These findings were different from Kopuz C, et al. [8] who recorded that $60 \%$ of the anastomosis was found between the main trunk \& the upper division. In the present study there was no any type of anastomosis identified in $20 \%$ of the cases. In 2014, Yang HM, et al. [11] carried out a study on the clinical implications of facial nerve topography and anastomosis in the condylar region, they concluded that the number of tiny branches and interconnections in the temporomandibular joint region is far less than those supply the facial muscles, so meticulous fine dissection in this region must be considered regardless the type or approach of the surgery.

Notably it is critical for the surgeons to have a basic and integrated knowledge about the morphometry and pattern branching of the facial nerve for a meticulous fine surgery in the parotid region. Decision making regarding selection of the type of the incision and technique in the parotid surgery is associated significantly to the origin of the buccal branch to avoid area of greatest vulnerability [12].

\section{Conclusion}

In most cases of the present study the buccal nerve branches originated from the lower division mainly as well as both divisions. There was similarity between both sides in the most common pattern of buccal nerve branch.

Bilateral facial nerve configuration was almost $50 \%$ for both similarities and differences. Most anastomotic interconnections were identified between the lower and upper division \& between the lower division and the buccal nerve branch.

\section{References}

1. Michael Monteiro (2008) Head and Neck. In: Standring S, Borley NR (eds) Gray's Anatomy: The Anatomical Basis of Clinical Practice. $40^{\text {th }}$ edition. Churchill Livingstone/Elsevier, 494-495.

2. Kwak HH, Park HD, Youn KH, Hu KS, Koh KS, et al. (2004) Branching Patterns of the Facial Nerve and its Communication with the Auriculotemporal Nerve. Surg Radiol Anat 26: 494-500.

3. Gataa IS, Faris BJ (2016) Patterns and Surgical Significance of Facial Nerve Branching Within the Parotid Gland in 43 Cases. Oral Maxillofac Surg 20: 161-165.

4. Mathieu Laurentjoye, Alice Veyret, Bruno Ella, André Pierre Uzel, Claire Majoufre-Lefebvre, et al. (2014) Surgical Anatomy of the Preauricular Anteroparotid Approach for Mandibular Condyle Surgery. Surg Radiol Anat 36: 883-888.

5. Kehrer A, Engelmann S, Ruewe M, Geis S, Taeger C, et al. (2019) Anatomical Study of the Zygomatic and Buccal Branches of the Facial Nerve: Application to Facial Reanimations Procedures. Clin Anat 32: 480-488.

6. Davis RA, Anson BJ, Budinger JM, Kurth LR (1956) Surgical Anatomy of the Facial Nerve and Parotid Gland Based Upon a Study of 350 Cervicofacial Halves. Surg Gynecol Obstet 102: 385-412.

7. Farooq Ahmed, Farooq Ahmed, Muhammed Ehsanul Haque, Riaz Ahmad, Imran Ahmed, et al. (2005) Facial Nerve Pattern of Distributions in Parotid Gland. Professional Med J 12: 85-90.

8. Kopuz C, Turgut S, Yavuz S, Ilgi S (1994) Distrbution of Facial Nerve in Parotid Gland; Analysis of 50 Cases. Okajimas Folia Anat Jpn 70: 295-299.

9. Katz AD, Catalano $P$ (1987) The Clinical Significance of the Various Anastomotic Branches of the Facial Nerve: Report of 100 Patients. Arch Otolaryngol Head Neck Surg 113: 959-962.

10. Yang HM, Won SY, Kim HJ, Hu KS, (2013) Sihler Staining Study of Anastomosis Between the Facial and Trigeminal Nerves in the Ocular Area and Its Clinical Implications. Muscle Nerve 48: 545-550.

11. Yang HM, Yoo YB (2014) Anatomy of the Facial Nerve at the Condylar Area: Measurement Study and Clinical Implications. Scientific World Journal.

12. Salasche SJ, Bernstein G, Senkarik M (1988) Surgical Anatomy of the Skin. Appleton \& Lange, University of Michigan 8-10. 